\title{
Time-Dependent Multiscale Simulations of Fire Emergencies in Longitudinally Ventilated Tunnels
}

\author{
FRANCESCO COLELLA ${ }^{1}$, GUILLERMO REIN ${ }^{2}$, VITTORIO VERDA ${ }^{1}$, ROMANO BORCHIELLINI ${ }^{1}$, \\ and JOSÉ L. TORERO ${ }^{2}$ \\ ${ }^{1}$ Dipartimento di Energetica \\ Politecnico di Torino, Italy \\ ${ }^{2}$ University of Edinburgh \\ BRE Centre for Fire Safety Engineering, UK
}

\begin{abstract}
This paper applies a novel and fast multiscale approach to model ventilation flows and fires in tunnels. The complexity and high cost of full CFD models and the inaccuracies of simplistic zone or analytical models are avoided by efficiently combining mono-dimensional (1-D) and CFD (3-D) modeling techniques. The multiscale model couples a 3-D CFD solver with a simple 1-D model allowing for a more rational use of the computational resources. The 1-D network models tunnel regions where the flow is fully developed (far field), and detailed CFD is used where flow conditions require 3-D resolution (near field). The multiscale method has been applied to model steady-state fires and tunnel ventilation systems, including jet fans, vertical shafts and portals, and it is applied here to study the transient flow interactions in a modern tunnel of $7 \mathrm{~m}$ diameter section and $1.2 \mathrm{~km}$ in length. Different ventilation scenarios are investigated to provide the timing to reach the critical velocity conditions at the seat of the fire and to remove the upstream back layering. The much lower computational cost is of great value, especially for parametric and sensitivity studies required in the design or assessment of real ventilation and fire safety systems. This is the first time that a comprehensive analysis of the transient fire and ventilation flow scenarios in a long tunnel is conducted.
\end{abstract}

KEYWORDS: tunnel fires, multiscale modeling, CFD, smoke management, detection.

\section{THE NEED FOR MULTISCALE MODELLING}

Fire disasters like Mont Blanc tunnel (Italy, 1999) and the more recent three fires in the Channel Tunnel (2008, 2006 and 1996) show that tunnel fire emergencies must be managed by a global safety system and strategies capable of integrating detection, ventilation, evacuation and firefighting response, keeping as low as possible damage to occupants, rescue teams and structures. Within this safety strategy, the ventilation system plays a crucial role because it takes charge of maintaining tenable conditions to allow safe evacuation and rescue procedures as well as fire fighting. The transient interaction between tunnel ventilation flows and fire dynamics is an important issue in tunnel fire protection.

The study of tunnel ventilation flows and fires is most economical and time efficient when done using computer models. The most computationally cheap models are 1-D models which were developed in the 1970s. These provide the overall behavior of the ventilation system under the assumptions that all the fluiddynamic quantities are effectively uniform in each tunnel cross section and gradients are only present in the longitudinal direction. Because 1-D models have low computational requirements, they are especially attractive, and are widely used by industry, for parametric studies where a large number of simulations is required.

Computational fluid dynamics (CFD) remains the most powerful method to predict the flow behavior due to ventilation devices, large obstructions or fire. In the last two decades, the application of CFD as a predictive tool for fire safety engineering has become widespread. However, CFD analysis of fire phenomena within tunnels suffers from the limitations set by the size of the computational domains and requires large computational resources. The computational cost escalates with the tunnel length and often becomes impractical for engineering purposes even for medium tunnels less than $500 \mathrm{~m}$ long. The task is near impossible for large tunnels (kilometers long).

Studies of ventilation and fire-induced flows in tunnels [1-3] pointed out that in the vicinity of operating jet fans or close to the fire source the flow field has a complex 3-D behavior with large transversal and longitudinal temperature and velocity gradients. The flow in these regions needs to be calculated using 
CFD tools since any other simpler approach would only lead to inaccurate results. These regions are hereafter named as the near field. However, it has been demonstrated for cold flow scenarios and for fire scenarios that some distance downstream of these regions, the temperature and velocity gradients in the transversal direction tend to disappear and the flow becomes essentially 1-D. In this portion of the domain the transversal components of the velocity can be up to two orders of magnitude smaller than the longitudinal components [3]. These regions are hereafter named as the far field. The use of CFD models to simulate the fluid behavior in the far field leads to large increases in the computational requirements but very small improvements in the accuracy of the results.

On the basis of these observations and for the sake of an efficient allocation of resources, CFD could be applied only to model the near field regions while the far field regions should be simulated using a 1-D model. These types of hybrid models are commonly called multiscale models. Multiscale models allow a significant reduction in the computational time as the more time consuming tool is applied only to a limited portion of the domain. Multiscale techniques have been applied to steady-state flow problems in long tunnels, for cold ventilation flows [1,4] and to fire induced flows [2]. In the first case the multiscale model has been used to simulate the jet fan discharge cone from jet fans. The predicted velocity values were compared with experimental measurements in a real tunnel [1,4]. It was demonstrated that the model can provide accurate results with a significant reduction of the computational requirements. The work has been applied to asses the performance of the Dartford Tunnels (London, UK) during emergency ventilation strategies and determine the level of redundancy [4]. These works demonstrated that multiscale modeling is a compelling technique for the simulation of complex tunnel ventilation systems, and furthermore, it represents the most feasible tool to conduct accurate simulations in long tunnels.

The current paper applies multiscale modeling for the first time to solve tunnel transient flows enabling for the efficient investigation of the dynamic interaction between the fire and the whole tunnel ventilation system.

\section{MULTISCALE MODELLING}

In a multiscale approach, the CFD and the 1-D models exchange flow information at the 1-D CFD interfaces. There are two general coupling options. The simplest one is the 1-way coupling (or superposition). For example, in the case of an inclined tunnel, it is possible to evaluate the global chimney effect using a 1-D model of the entire tunnel [5]. Then, a CFD analysis of specific tunnel portions can be run using as boundary conditions the 1-D results. This approach does not couple the CFD to the 1-D model and assumes that the flow behavior in the high-gradient regions does not affect the bulk tunnel flow.

A 2-way coupling of 1-D and CFD models, a proper multiscale modeling consists of a physical decomposition of the domain in two or more sub-domains (see Fig. 1): $\Omega_{3 D}$ represents the sub-domain modeled by means of CFD while a 1-D model is used in $\Omega_{1 D}$. If the solver is able to receive the two sets of equations, the problem can be solved at once. In most of the cases there is a different solver for each model, and therefore iterative calculations are necessary with the two solvers continuously exchanging information at boundary interfaces.

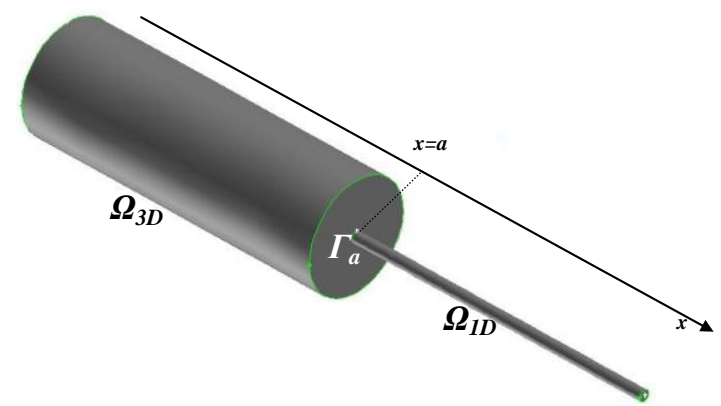

Fig. 1. Example of domain decomposition in 1-D and 3-D sub-domains. 


\section{The CFD Sub-Model}

This section describes the particular modeling of the near field conducted here using the commercial CFD code FLUENT. The fluid behavior in the 3-D sub-domain has been modeled using the classical Reynoldsaveraged Navier-Stokes equations (RANS) complemented with the k- $\varepsilon$ model for turbulence [6]. Fire induced flows are essentially driven by buoyancy forces which have a non-negligible impact on their turbulent nature. Therefore, the standard $\mathrm{k}-\varepsilon$ model, developed for constant density flows, has been modified to account for the production and the destruction of turbulent kinetic energy due to the large density gradients [7]. Such approach has been extensively used and largely validated by the scientific community to model fire induced flows $[3,8,9,11]$.

The fire has been modeled as a volumetric source of heat without a dedicated combustion model as used in Refs.[2] and [14]. The fire source is introduced into the computational domain as a rectangular slab releasing hot gases from the top surface simulating a burning vehicle. Mass conservation is applied by the extraction of air at the obstruction for lateral surfaces simulating air entrainment. The amount of combustion gases injected into the domain and the size of the slab are calculated using Froude scaling allowing for the correct balance between the momentum and buoyancy strength of the fire source. This simplified approach, previously used to model fire induced flows in tunnels $[12,13]$, is the most practical given its low computational cost. It avoids the burden and the uncertainty of combustion and radiation models and the large unknown associated to the burning of a mixture of condensed-phase fuels. It has been shown to provide good thermal and flow results away from the fire.

Quasi-structured meshing is used and, the grid has been systematically refined in order to assess whether or not a grid-independent solution was reached. The degree of convergence of the solution has been estimated by using scaled residual and by monitoring integral values of relevant quantities (typically mass flow rate through tunnel portals) during the solution procedure. The simulations have been considered to be converged when the scaled residuals were lower than $10^{-6}$ with the exception of the energy equation where the maximum allowed value was $10^{-8}$.

\section{The 1-D Sub-Model}

The general methodology of 1-D network models for tunnels is presented in $[1,2,14]$ and only a brief overview is given here. The developed 1-D model is based on a generalized Bernoulli formulation. It is designed to account for buoyancy effects, transient fluid-dynamic and thermal phenomena, piston effects and transport of pollutant species. It is developed to handle complex layouts typical of modern tunnel ventilation system (especially true for transverse ventilated tunnel) on the basis of a topological representation of the tunnel network. The fluid dynamic model needs the mass, momentum and energy conservation equations to be solved in the whole domain.

The 1-D problem can be solved after discretizing the computational domain in control volumes which allows the integration of momentum, continuity and energy equations. Typically, the tunnel domain is discretized in oriented elements called branches, interconnected by nodes. More details on the subject including modeling aspects and numerical features and are available in [14].

\section{Interaction Between 1-D and 3-D Sub-Models}

Multiscale techniques are typically based on domain decomposition methods which have been developed for all the discretization techniques (i.e. finite difference, finite volume and finite elements) mainly in the framework of parallel computing. They allow the original single problem to be reformulated on several computational sub-domains. Eventually, this technique can be applied to solve heterogeneous problems which are described by different governing equations as in the present case.

The basic idea is to decompose the global domain into several sub-domains and to solve the resulting problems characterized by smaller domain size. The exact structure of the boundary conditions to be applied at the interfaces depends on the differential operator defining the original set partial differential equations and by the presence of overlapping domain regions. In the case of Navier-Stokes equations two iterative methods are used: Dirichlet-Dirichlet (or Schwartz) and Dirichlet-Neumann [15]. DirichletDirichlet coupling requires the definition of overlapping regions between 1-D and 3-D sub-domains and its 
application is cumbersome. The iterative Dirichlet-Neumann does not require the presence of overlapping regions and is easier to implement in FLUENT. This is the approach used here.

The iterative solving algorithm is presented for a general case in which a CFD model of the near field domain $\left(\Omega_{3 D}\right)$ is coupled with two 1-D models of far field domains $\left(\Omega_{1, I D}\right.$ and $\left.\Omega_{2, I D}\right)$ located upstream and downstream, respectively. Two interfaces $\Gamma_{i}$ and $\Gamma_{j}$ are therefore generated (see Fig. 2.).

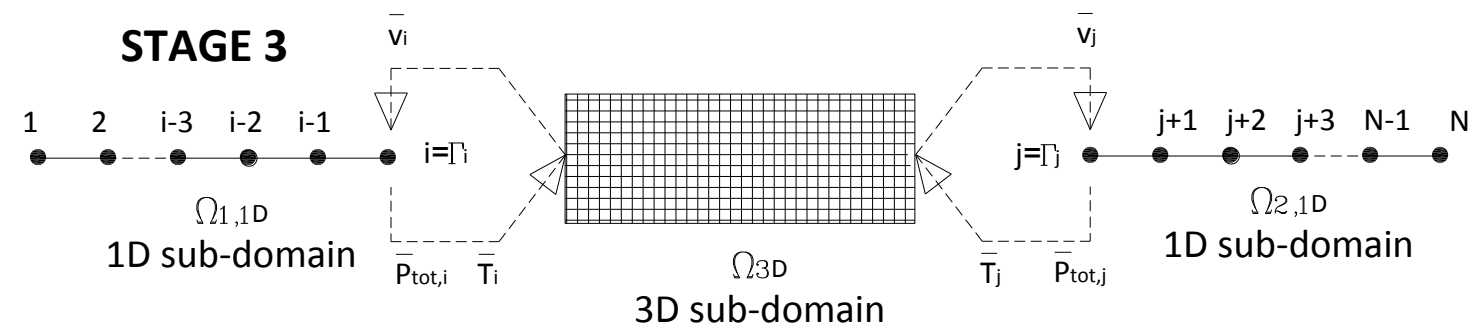

Fig. 2. Visualization of the coupling procedure.

Following the same approach presented in Ref. [16], pressure, velocity and temperature continuity at the 1-D-3-D interfaces is enforced. As shown in Ref. [16], imposing the continuity of the averaged normal stresses is equivalent to imposing the continuity of the mean pressure. This is adequate because the normal stresses are partially neglected in the 1-D model, and the interfaces are located in regions where the flow is fully developed (thus, $\partial u / \partial x \approx 0$ ). Nonetheless, the validity of this assumption is checked in each multiscale computation.

The solution of the multiscale problem is reached by means of iterative computing procedures, known also as interaction model, developed in the framework of domain decomposition methods. This iterative procedure allows the 1-D and CFD models to interact dynamically during the solution procedure by periodically exchanging the boundary conditions at the interface. More details on the subject can be found in Ref. [14].

The interaction model used here is split into two sub-problems

- A 1-D problem of the far fields $\Omega_{l, I D}$ and $\Omega_{2, I D}$ with Dirichlet boundary conditions (velocity boundary conditions) at the interfaces

- A 3-D problem of the near field $\Omega_{3 D}$ with Neumann boundary conditions (i.e. pressure boundary conditions) at the interfaces

The global multiscale convergence is reached by iterating $k$-times between the two sub-problems until a global solution is obtained.

\section{CASE STUDY: MODERN TUNNEL 1.2 KM LONG}

The multiscale technique outlines above has been used to simulate the transient flows generated from the coupling of fire dynamics to the ventilation system in a modern generic tunnel. A schematic of the specific tunnel layout is presented in Fig. 3. It is a $1200 \mathrm{~m}$ long tunnel, $6.5 \mathrm{~m}$ high with standard horseshoe cross section of around $53 \mathrm{~m}^{2}$ and hydraulic diameter around $7.3 \mathrm{~m}$. The tunnel is longitudinally ventilated and equipped with two groups of 5 jet fans pairs $50 \mathrm{~m}$ spaced, each group installed near a tunnel portal. The jet fans are rated by the manufacturer at the volumetric flow rate of $8.9 \mathrm{~m}^{3} / \mathrm{s}$ with a discharge flow velocity of $34 \mathrm{~m} / \mathrm{s}$. All vehicles are assumed to have left the tunnel safely and no obstacles remain. 


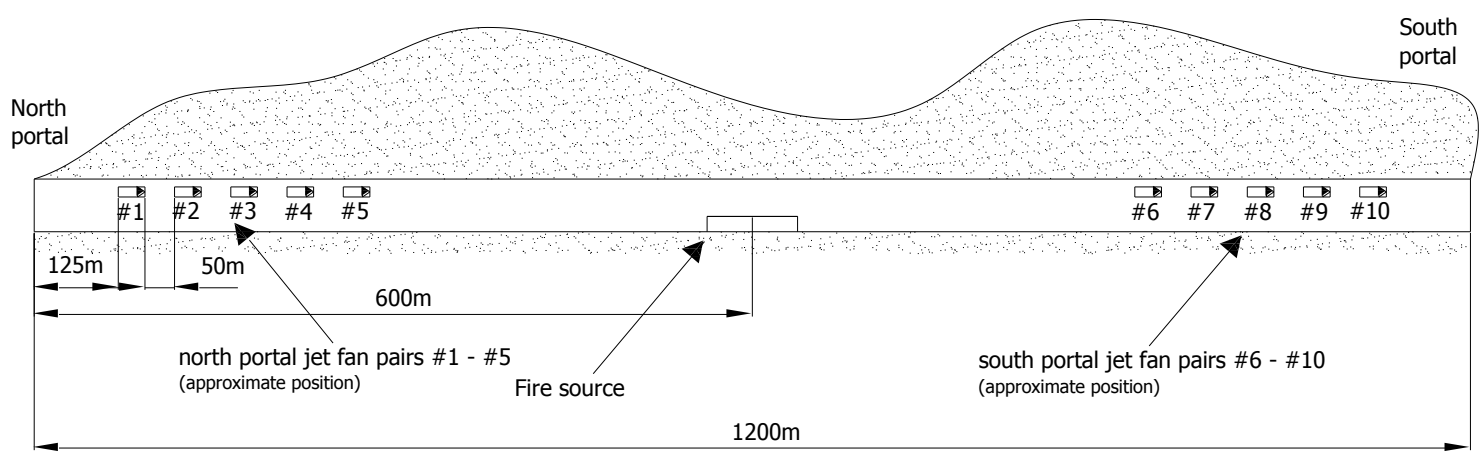

Fig. 3. Layout of the tunnel used as case study showing the relative positions of the fire, jet fans and portals. (not to scale)

The emergency ventilation strategy, as for most longitudinally ventilated tunnels, requires the ventilation system to push all the smoke downstream from the incident region, thus avoiding the back-layering effect when smoke spreads against the ventilation flow. Also, the upstream region should be cleared of smoke as soon as possible to allow for safe evacuation and fighting activities. Thus, an adequate ventilation system has to provide air velocities higher than the critical velocity at the location of the fire. Studies on back layering show that the maximum critical velocity is in the range from $2.5 \mathrm{~m} / \mathrm{s}$ to $3 \mathrm{~m} / \mathrm{s}[10,11,17]$.

\section{Grid Independence Study}

The computational domain has been discretized using quasi structured meshes with refinements introduced close to the walls and in the vicinity of the fire source. Various full CFD runs have been conducted to estimate the mesh requirements. Four different meshes characterized by densities ranging from $100 \mathrm{cells} / \mathrm{m}$ to 4000 cells/m were generated and the resulting solutions compared (see Fig. 4). The study confirmed that a grid independent solution could be obtained when the mesh density is above 2500 cells $/ \mathrm{m}$. An example of CFD solutions computed over the four different mesh patters is presented in Fig. 5. and refers to a tunnel cross section located $100 \mathrm{~m}$ downstream of the fire source. As expected, the computed solutions show larger deviations for the coarse meshes 1 and 2 while convergence of the temperature and velocity fields is obtained for finer meshes 3 and 4. Based on the results, grid independence is considered reached for mesh 3 and therefore, all the following simulations have been conducted using this mesh. More data and details on issue can be found in [2]. The mesh densities providing grid independent results depend on the particular tunnel geometry, ventilation and fire scenario. Therefore, it is recommended to conduct grid independence analysis when different tunnels and fire scenarios are studied.

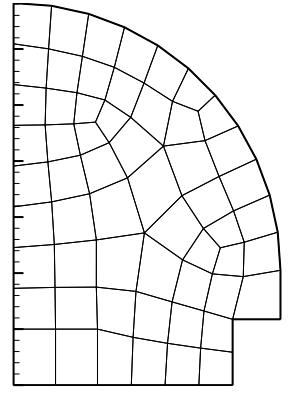

Mesh 1 105 cells $/ \mathrm{m}$

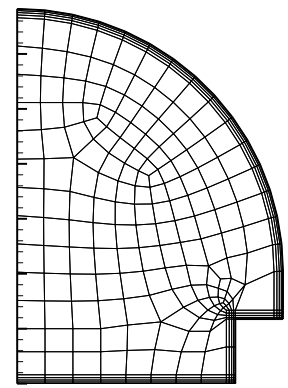

Mesh 2 695 cells $/ \mathrm{m}$

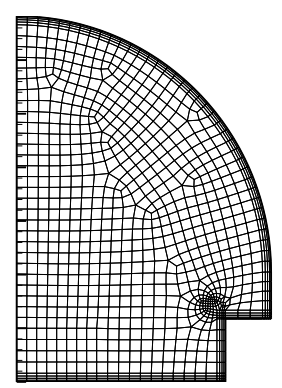

Mesh 3 2525 cells $/ \mathrm{m}$

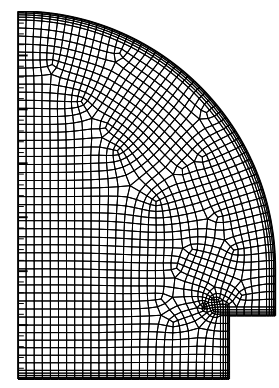

Mesh 4 $4125 \mathrm{cells} / \mathrm{m}$

Fig. 4. Examples of the different meshes used for half of the tunnel cross section and number of cells per unit length of tunnel. 

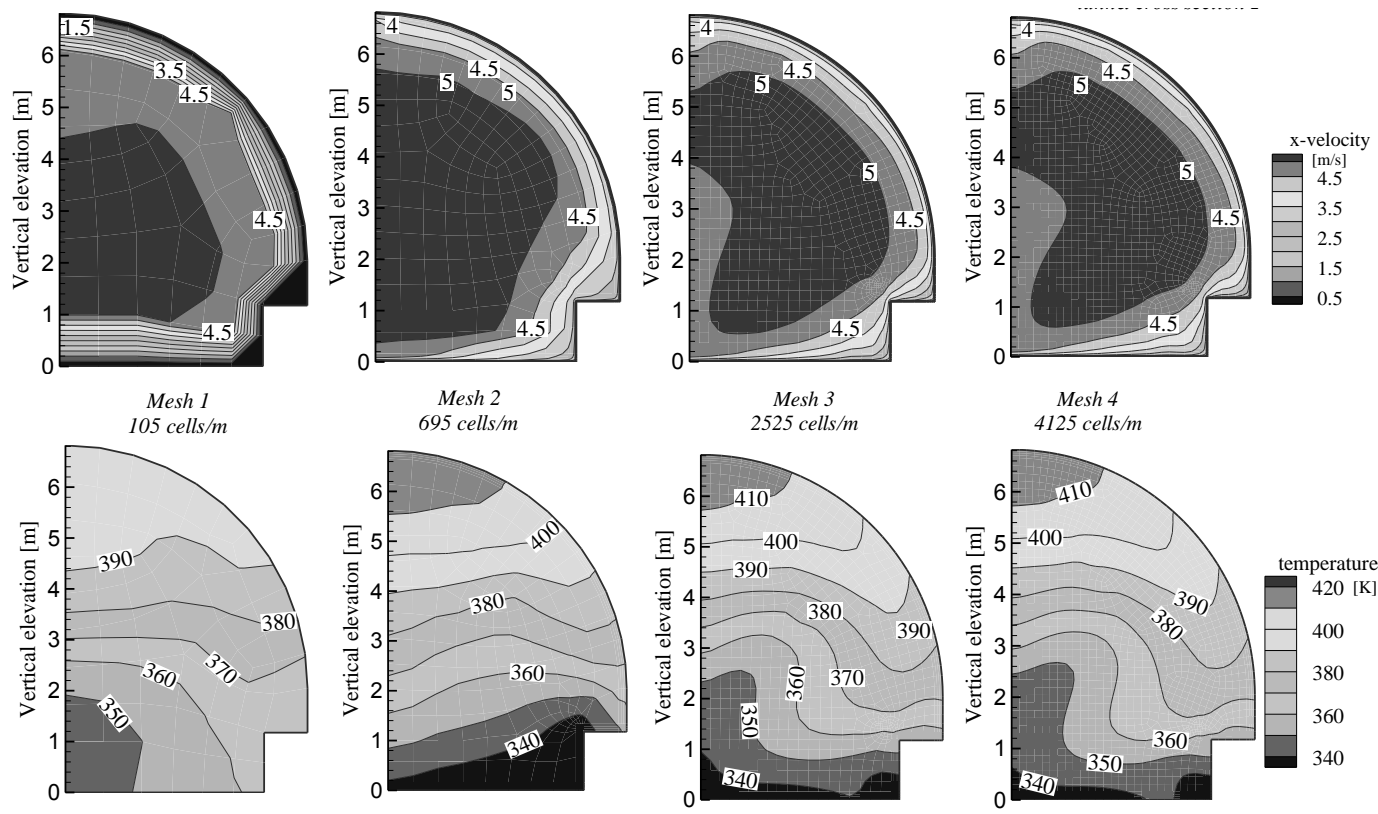

Fig. 5. Comparison of the longitudinal velocity (top) and temperature (bottom) contours for meshes \#1 to $\# 4$ in the tunnel at tunnel reference section located $100 \mathrm{~m}$ downstream of a $30 \mathrm{MW}$ fire. The velocity and temperature values are expressed in $\mathrm{m} / \mathrm{s}$ and $\mathrm{K}$, respectively.

\section{Boundary Independence Study}

A schematic of the coupling between 1-D model of the far field and CFD model of the near field has been depicted in Fig. 6.

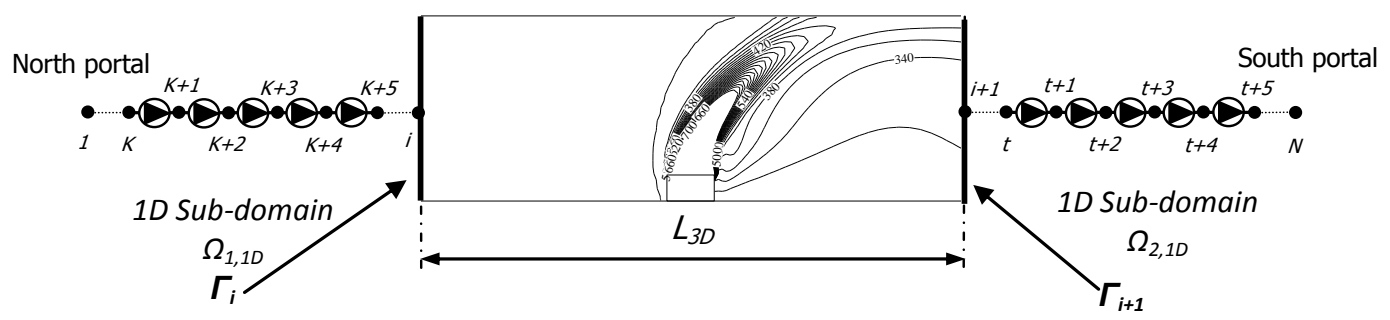

Fig. 6. Schematic of the multiscale model of the $1.2 \mathrm{~km}$ tunnel from portal to portal and including 10 jet fans pairs. The CFD domain of the fire region contains temperature contours showing the fire plume. The 1-D CFD interfaces are highlighted. (not to scale)

An important element in multiscale modeling is the correct positioning of the 1-D-3-D interfaces $\left(\Gamma_{i}\right.$ and $\Gamma_{i+1}$ in Fig. 6). These must be located in a region where the flow is fully developed (i.e. 1-D flow) and characterized by mild longitudinal velocity gradients.

A study has been performed in order to identify the dependence of the local flow field solutions on the position of the 1-D-3-D interfaces and consequently on the dimension of the CFD domain ( $L_{3 D}$ in Fig. 6). Four different fire sizes ranging from $10 \mathrm{MW}$ to $100 \mathrm{MW}$ have been considered for the study. Flow field data obtained from full CFD simulations have been taken as reference solution. The comparison has been performed on two different reference cross-sections located $10 \mathrm{~m}$ and $100 \mathrm{~m}$ downstream of the fire source. For sake of simplicity, the results, expressed as relative deviation from the full CFD solutions, are presented in Fig. 7 only for the reference section located $10 \mathrm{~m}$ downstream of the fire source. 

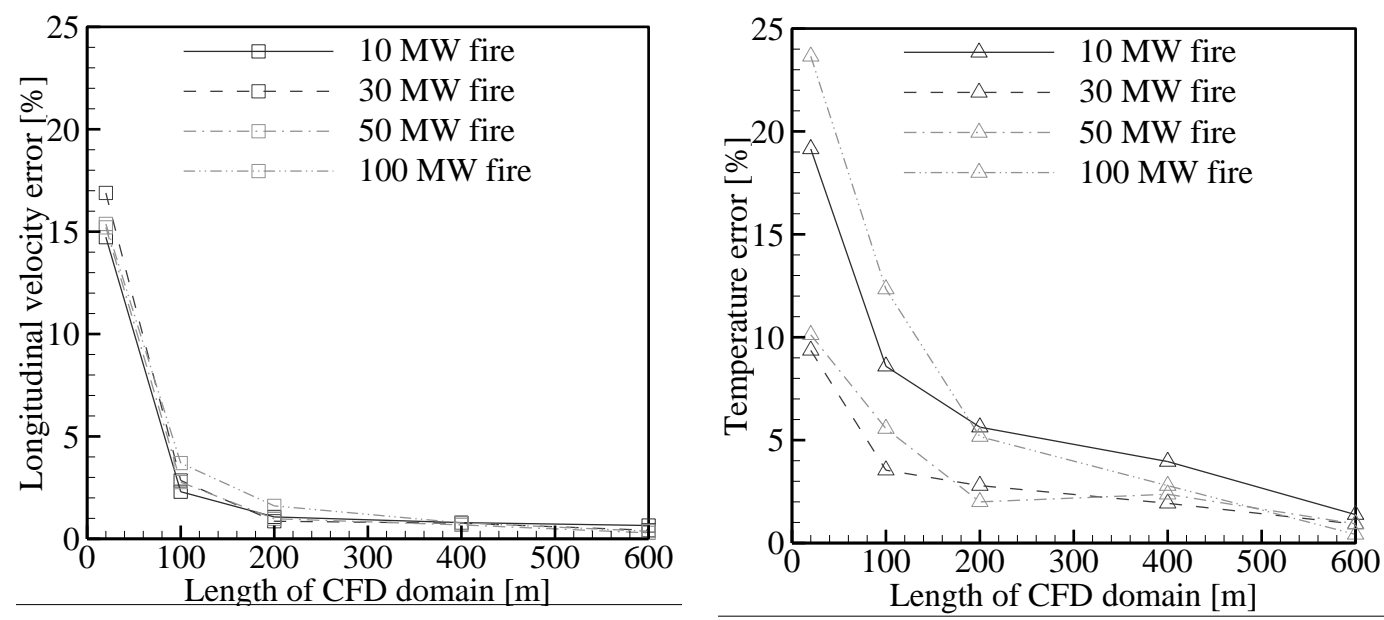

Fig. 7. Effect of the CFD domain length $L_{3 D}$ on the error for the average longitudinal velocity and average temperature. Results compared at a reference section located $10 \mathrm{~m}$ downstream of the fire source.

The results show that the error does not depend significantly on the dimension of the fire within that range. The solution for a $20 \mathrm{~m}$ long domain provides low accuracy (15\% error). The results become boundary independent and provide less than a few percents error for domain lengths larger than $200 \mathrm{~m}$. The complete analysis, which can be found in Ref. [2], confirms that if the downstream boundary is located at a distance from the fire larger than 13 times the tunnel hydraulic diameters the multiscale solution in the vicinity of the fire source matches perfectly the full-CFD solution.

On the basis of this estimation, the outlet boundary in this work has been located at distance equal to 20 times the tunnel diameter $(\sim 150 \mathrm{~m})$ and therefore, the length of the CFD sub-domain is equal to $300 \mathrm{~m}$ being the fire located in the middle.

The assumption of fully developed flow conditions at the upstream boundary must be maintained during the whole calculation process. This is most challenging during the initial stages after fire ignition, when the ventilation system has not yet been activated or the airflows are still sub-critical. The back-layering nose will travel towards the upstream 1-D-3-D interface and it must be ensured that it does not cross it. If otherwise, there will be a tunnel region close to the upstream boundary where the computed flow field will present deviation from the full-CFD solution as discussed for the downstream boundary.

A rough estimation of the smoke front velocity in the absence of forced ventilation can be obtained based empirical correlations [18]. However, a posteriori check of the results to confirm that the back-layering nose is contained within the CFD module during sub-critical periods must always be conducted.

\section{Steady-State Results}

The results are relative to three different ventilation scenarios involving a $30 \mathrm{MW}$ fire located in the middle of the tunnel and 3,5 and 10 operating jet fan pairs, respectively. The accuracy of the computed multiscale solution was assessed by comparing both flow field data and integral values to data from full CFD calculations.

The first comparison, relative to Scenario 1, is presented in Fig. 8 which plots temperature and horizontal velocity fields on the tunnel longitudinal plane both for multiscale and full CFD calculations. The multiscale model predictions compare very well to the full CFD predictions. In particular no appreciable differences are observed in the temperature field. Very small differences are observed in the longitudinal velocity field. These small differences are due to the presence of the discharge cone generated by the operating jet fans upstream of the fire source which are included in the full CFD representation. 


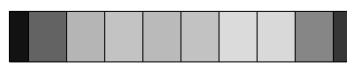

temperature: $325 \quad 375 \quad 425 \quad 500 \quad 600[\mathrm{~K}]$

X-velocity: $\quad-3 \quad-1 \quad 1 \quad 3 \quad 3 \quad 5 \quad 7 \quad[\mathrm{~m} / \mathrm{s}]$

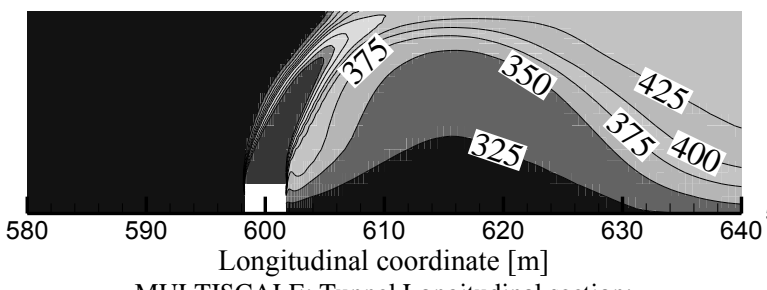

MULTISCALE: Tunnel Longitudinal section:
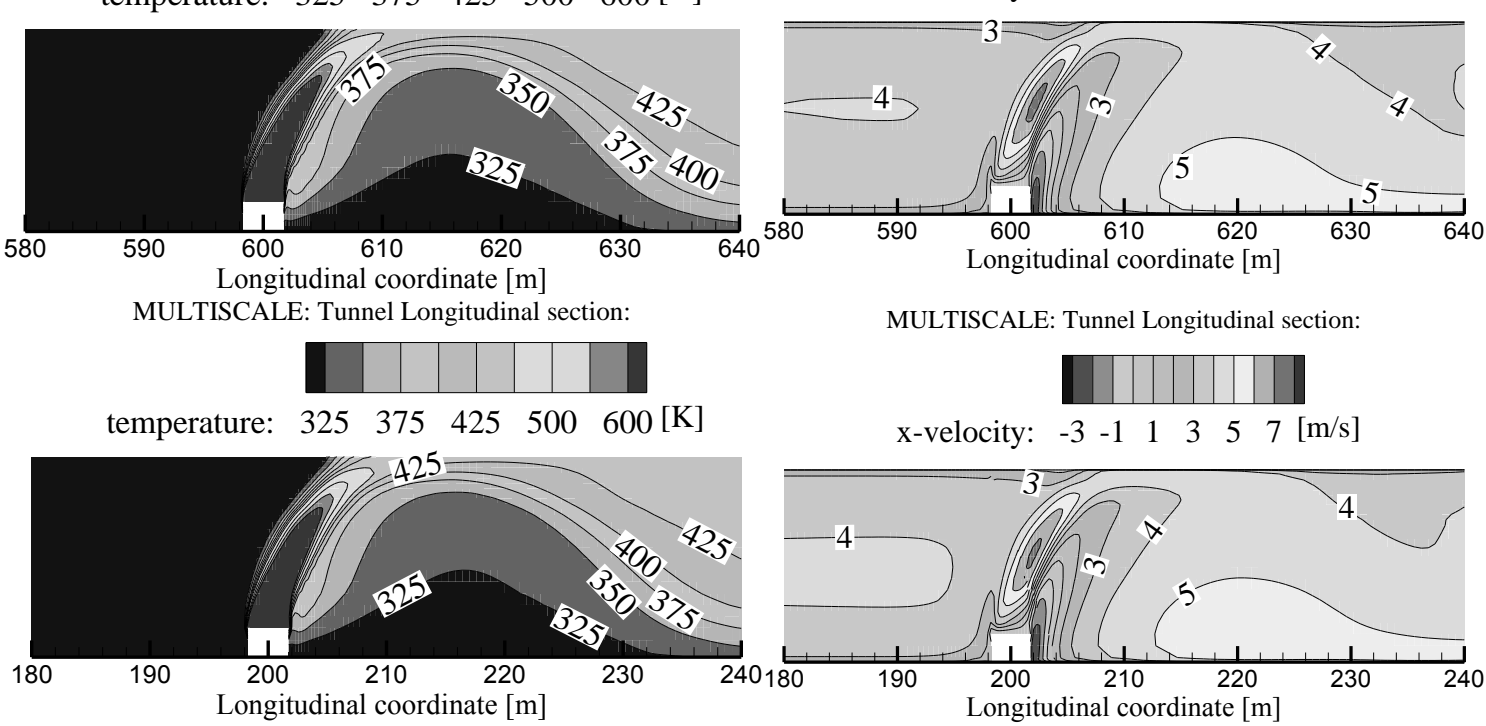

MULTISCALE: Tunnel Longitudinal section:

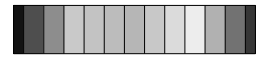

x-velocity: $\quad-3 \quad-1 \quad 1 \quad 3 \quad 3 \quad 5 \quad 7[\mathrm{~m} / \mathrm{s}]$

Fig. 8. Comparison of results near the fire for the multiscale and the full CFD simulations for a fire of $30 \mathrm{MW}$ and ventilation Scenario 1 . Velocity and temperature values are expressed in $\mathrm{m} / \mathrm{s}$ and K respectively. The longitudinal coordinates start at the upstream boundary of the corresponding CFD domain.

The deviation of integral values for the mass flow rate through the tunnel is smaller than $2 \%$ in all the scenarios involving a $30 \mathrm{MW}$ fires (see Table 1). Moreover, it must be stressed that the multiscale calculations required significantly lower computational times $(2 \mathrm{~h}$ vs. between $48 \mathrm{~h}$ and $72 \mathrm{~h}$ for the full CFD). More scenarios involving different fire sizes and ventilation scenarios can be found in Ref. [2].

Table 1. Summary of the ventilation scenarios for the steady state analysis and accuracy of the multiscale simulations.

\begin{tabular}{|c|c|c|c|c|c|c|c|}
\hline Scenario & Fire & Jet fan & Jet fan & Jet fan & Full CFD & \multicolumn{2}{|c|}{ Multiscale } \\
\cline { 6 - 8 } & size & pairs & pair & pairs & Mass flow & Mass flow & Deviation \\
& (MW) & $\# \mathbf{- 3}$ & $\mathbf{\# 4 - 5}$ & $\mathbf{\# 6 - 1 0}$ & rate (kg/s) & rate (kg/s) & \\
\hline $\mathbf{1}$ & 30 & $\underline{\text { ON }}$ & OFF & OFF & 216 & 220 & $2.09 \%$ \\
\hline $\mathbf{2}$ & 30 & $\underline{\text { ON }}$ & $\underline{\text { ON }}$ & OFF & 301 & 301 & $0.01 \%$ \\
\hline $\mathbf{3}$ & 30 & $\underline{\text { ON }}$ & $\underline{\text { ON }}$ & $\underline{\text { ON }}$ & 435 & 434 & $0.15 \%$ \\
\hline
\end{tabular}

\section{Transient Results}

The time sequence of event is the following. At time $t=0$, a fire is ignited and start growing. After a certain time (time to detection, TD), the fire detection system is triggered and then the ventilation strategy is activated. The airflow in the tunnel accelerates and drives the smoke towards the South portal. When supercritical ventilation velocities are reached, the back-layering starts to retreat towards the seat of the fire. Once the upstream region of the tunnel is totally free of smoke, it is safe for evacuation and firefighting procedures to take places.

The fire is assumed to take place in middle of the tunnel and reach a peak value of $30 \mathrm{MW}$. After ignition, the power of the fire (the heat release rate, HRR) grows as shown in Fig. 9. The fire growth curve has been built on the basis of the work by Carvel [19] applicable to typical fires of European HGVs cargos with a two-step linear growth instead. During the first regime, the fire grows slowly up to 1 or 2 MW at 4 to $14 \mathrm{~kW} / \mathrm{s}$, and after a delay typically lasting from 120 to $360 \mathrm{~s}$, the fire transitions to a much faster growth 
regime at 30 to $400 \mathrm{~kW} / \mathrm{s}$. For this case study, average values have been chosen; the slow regime grows at $8 \mathrm{~kW} / \mathrm{s}$, the delay is $240 \mathrm{~s} \mathrm{(4} \mathrm{min)} \mathrm{long,} \mathrm{and} \mathrm{the} \mathrm{fast} \mathrm{regime} \mathrm{grows} \mathrm{at} 250 \mathrm{~kW} / \mathrm{s}$. Hence, the peak power of $30 \mathrm{MW}$ is reached $350 \mathrm{~s}$ after ignition and remains at the peak value indefinitely during the simulation.

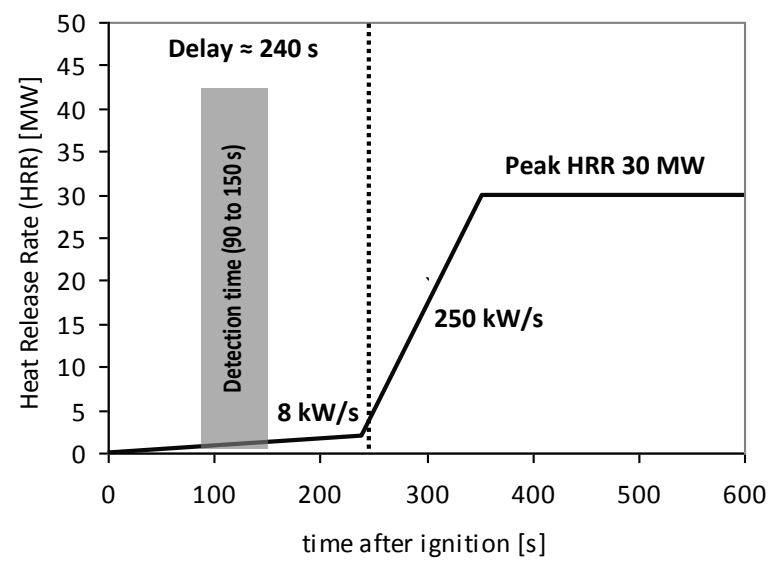

Fig. 9. Evolution of the growing fire (heat release rate) after ignition and time to detection considered in the transient simulations. The two-step fire growth curve is based on the work of Carvel (2008) [19].

In general, the time to detection (TD) depends on the technology used, the initial ventilation flows and the fire growth rate. It is approximately $120 \mathrm{~s}$ for typical slow growing fire in a tunnel equipped with fiber optic linear detection cables [20], which is one of the most popular detection technologies in modern tunnels. Shorter detection times could be expected for more efficient detection techniques (i.e. thermal imaging [21]) or faster growing fires (i.e. pool fires). And longer detection times could be expected if the fire is shielded by obstacles (e.g. wreck), or located inside or underneath the vehicle [22].

The same three ventilation strategies used for the steady state analysis have been used for the time dependent analysis too (see Table 2): Scenario 1 with operating jet fans pairs from \#1 to \#3 (6 jet fans); Scenario 2 with operating jet fans pairs from \#1 to \#5 (10 jet fans); Scenario 3 with operating jet fans pairs from \#1 to \#10 (all 20 jet fans). Each operating jet fan is activated right after the fire is detected and is assumed to reach full thrust $10 \mathrm{~s}$ after activation, as for typical modern devices. The detection time has been kept constant equal to $120 \mathrm{~s}$. The simulations are run until steady-state flow conditions in the tunnel are reached, being this less than $10 \mathrm{~min}$ in all simulations presented

Table 2. Summary of the ventilation scenarios considered in the time-dependent analysis and summary of the main findings.

\begin{tabular}{|c|c|c|c|c|c|c|c|}
\hline Scenario & $\begin{array}{c}\text { Fire } \\
\text { size } \\
\text { (MW) }\end{array}$ & $\begin{array}{c}\text { Jet fan } \\
\text { pairs } \\
\# \mathbf{1}-\mathbf{3}\end{array}$ & $\begin{array}{c}\text { Jet fan } \\
\text { pair } \\
\mathbf{\# 4 - 5}\end{array}$ & $\begin{array}{c}\text { Jet fan } \\
\text { pairs } \\
\# \mathbf{6}-\mathbf{1 0}\end{array}$ & $\begin{array}{c}\text { Detection } \\
\text { time (TD) } \\
\text { (s) }\end{array}$ & $\begin{array}{c}\text { Time to } \\
\text { critical } \\
\text { velocity (TC) } \\
\text { (s) }\end{array}$ & $\begin{array}{c}\text { Time to } \\
\text { remove back- } \\
\text { layering (TB) } \\
\text { (s) }\end{array}$ \\
\hline $\mathbf{1}$ & 30 & $\underline{\text { ON }}$ & OFF & OFF & 120 & 244 & 255 \\
\hline $\mathbf{2}$ & 30 & $\underline{\text { ON }}$ & $\underline{\text { ON }}$ & OFF & 120 & 190 & 220 \\
\hline $\mathbf{3}$ & 30 & $\underline{\text { ON }}$ & $\underline{\text { ON }}$ & ON & 120 & 156 & 187 \\
\hline
\end{tabular}




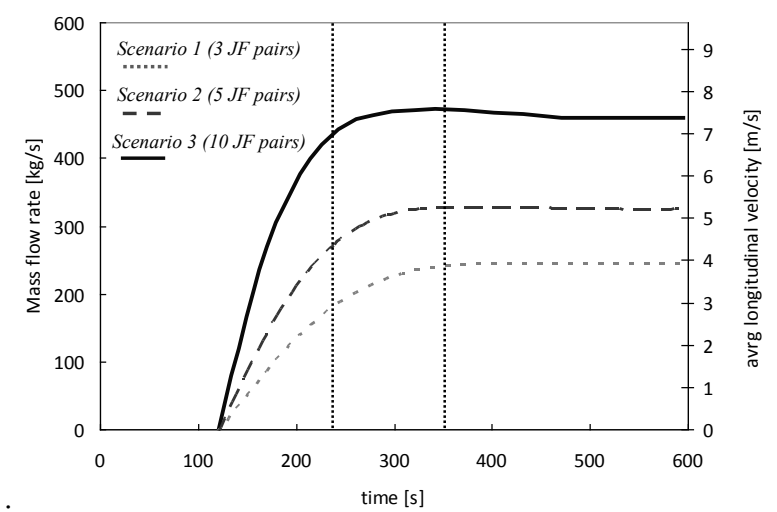

Fig. 10. Time dependent evolution of the mass flow rate through the tunnel for Scenarios \#1, \#2 and \#3 (see Table 2). The time to detection is $2 \mathrm{~min}$. Supercritical conditions $\left(v_{\text {air }}>3 \mathrm{~m} / \mathrm{s}\right)$ are reached after $244 \mathrm{~s}, 190 \mathrm{~s}$ and $160 \mathrm{~s}$ for Scenarios \#1, \#2 and \#3 respectively

Figure 10 shows the temporal evolution of the mass flow rate through the tunnel as computed by the multiscale model for the first three scenarios in Table 2 with a TD of 2 min. Supercritical ventilation conditions, corresponding to bulk flow velocity larger than $3 \mathrm{~m} / \mathrm{s}$, are reached $244 \mathrm{~s}, 190 \mathrm{~s}$ and $156 \mathrm{~s}$ after ignition (124 s, $70 \mathrm{~s}$ and $36 \mathrm{~s}$ after fire is detected and ventilation activated) for Scenario \#1, \#2 and \#3, respectively (see Table 2). At this moment in time the fire is still in the slow growth regime (except for Scenario \#1 for which the fire has just started the fast growth) and the HRR is lower than 2 MW. Steadystate flow conditions are reached in the tunnel at $380 \mathrm{~s}$ for Scenario \#1, that is $10 \mathrm{~s}$ after the fire has reached the peak HRR of $30 \mathrm{MW}$. For Scenarios \#2 and \#3, steady-state is reached before the peak HRR at 360 and $300 \mathrm{~s}$, respectively. Scenario \#3 shows a small tip at $350 \mathrm{~s}$ where a smooth transition from a growing fire to a steady fire takes place.

Figure 11 presents the computed temperature fields $300 \mathrm{~s}$ (top) and $600 \mathrm{~s}$ (bottom) after ignition for ventilation Scenarios from \#1 to \#3. The temperature contours computed $300 \mathrm{~s}$ after the ignition show that the back-layering has been removed in the three ventilation scenarios and that the smoke front is located at a distance of $350 \mathrm{~m}$ downstream of the seat of the fire for Scenario \#1 and $450 \mathrm{~m}$ for Scenario \#2. Since the ventilation velocities in Scenario \#3 are considerably higher, the smoke front has already reached the South portal and smoke is being released to the open atmosphere. The results show that smoke stratification downstream of the fire is lost at distances of 30,60 and $100 \mathrm{~m}$ for each scenario respectively. Therefore, only the tunnel regions upstream of the fire could be used for safe evacuation purposes. The average longitudinal velocity at the seat of the fire is approximately $3.5 \mathrm{~m} / \mathrm{s}, 5 \mathrm{~m} / \mathrm{s}$ and $7 \mathrm{~m} / \mathrm{s}$ for ventilation Scenarios \#1, \#2 and \#3, respectively.

Temperature fields within the tunnel domain $600 \mathrm{~s}$ after ignition show that both steady-state conditions have been reached and that smoke is being released to the atmosphere from the South portal for all scenarios. The average longitudinal velocity at the seat of the fire at $600 \mathrm{~s}$ is approximately $4 \mathrm{~m} / \mathrm{s}, 5.5 \mathrm{~m} / \mathrm{s}$ and $7.5 \mathrm{~m} / \mathrm{s}$ for ventilation each scenario respectively. 

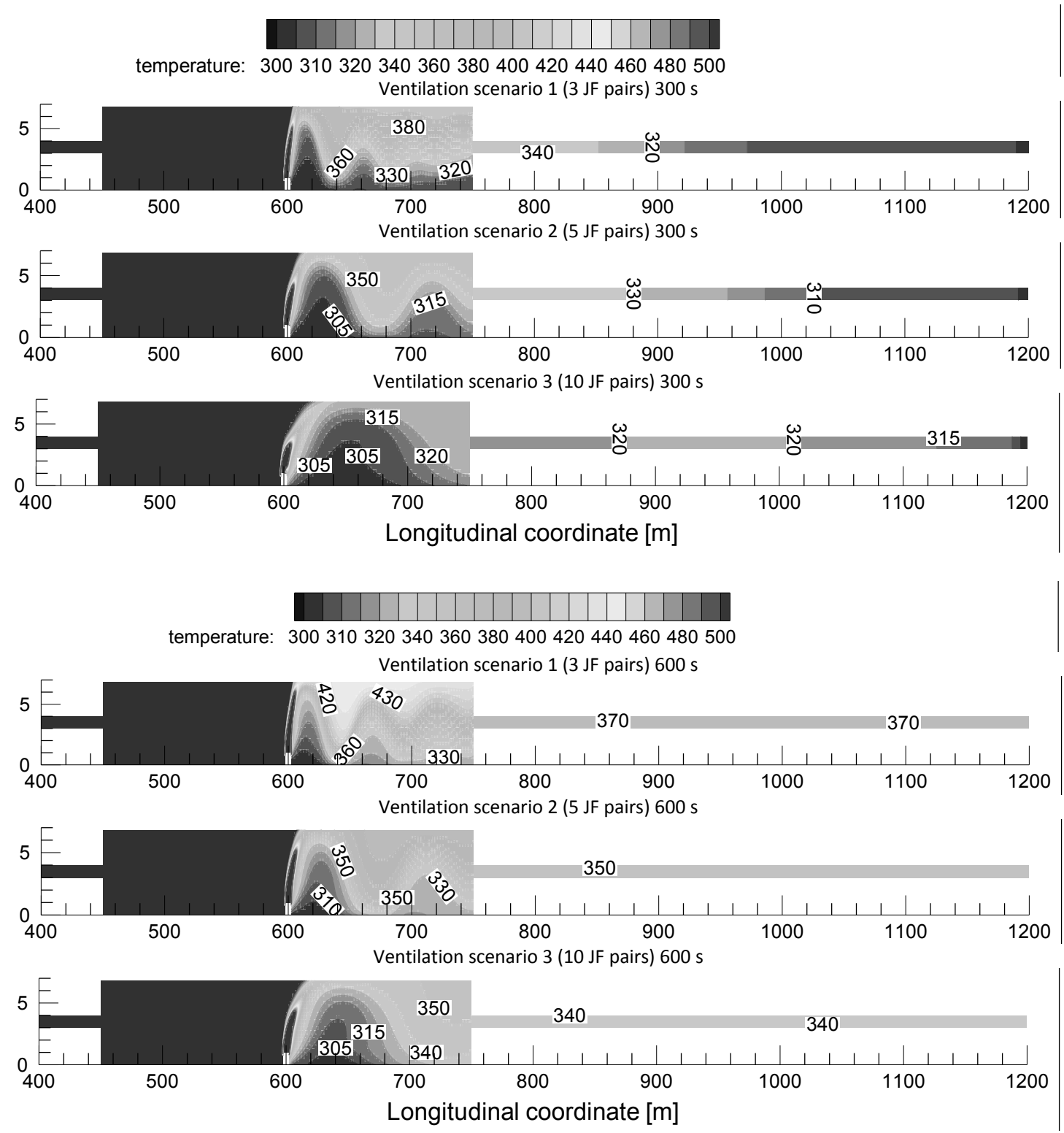

Fig. 11. Temperature fields $300 \mathrm{~s}$ (top) and $600 \mathrm{~s}$ (bottom) after ignition for Scenarios $\# 1, \# 2$ and $\# 3$ (see Table 2). Temperature values are expressed in $\mathrm{K}$. (not to scale)

There are two events of importance for the fire strategy taking place during a fire emergency. These are the time required to reach supercritical ventilation velocities in the fire region (TC), and the time required to remove the back-layering upstream from the fire (TB) Both are measured from the instant of fire ignition $(t=0)$, and it is desirable to minimize these so to create a safe passage free of smoke as soon as possible.

The analysis of these results allows for the assessment of the impact of the number of activated jet fans. The simultaneous activation of 10 jet fan pairs (Scenario \#3) compared to 3 jet fan pairs (Scenario \#1) reduces TC and TB by $36 \%$ and $30 \%$, respectively. The time to both critical events, either measured from ignition time or from detection time, decreases significantly as the number of activated jet fans increases.

Further simulations have been conducted to assess the impact of TD on the development of the emergency scenario within the tunnel. In particular, the detection time has been first increased to $150 \mathrm{~s}$ and then decreased to $90 \mathrm{~s}$. Figure 12 summarizes the findings on the impact of TD on the time required to reach critical velocity in the tunnel. 


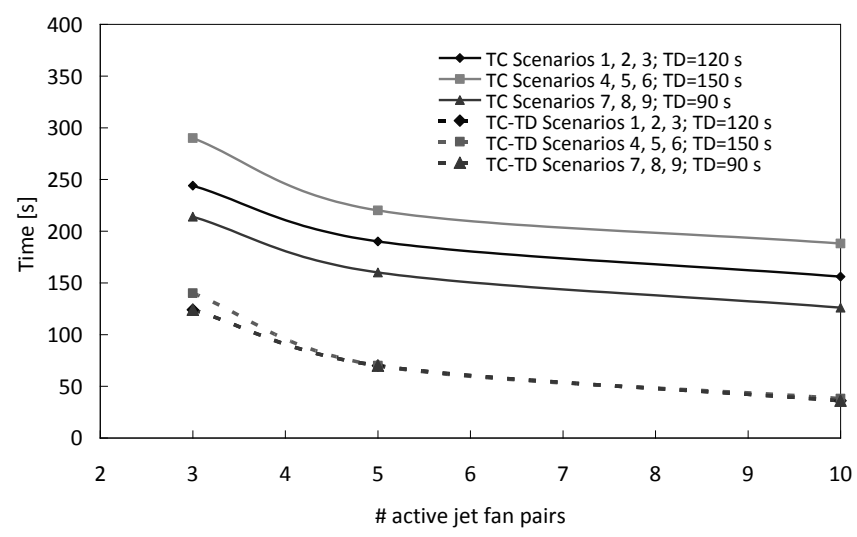

Fig. 12. Dependence of TC-TD (time required to remove back-layering after time to detection) and TC on the number of operating jet fan pairs and TD.

The results contained in Fig. 12 confirm that the values of (TC-TD) are dominated by the number of operating jet fans and that variations in TD only produce a simple shift of TC. This is explained because the ventilation system is able to generate supercritical velocities before the fire has reached a considerable size. For the fastest response case (TD equal to $90 \mathrm{~s}$ and 10 operating jet fan pairs) the fire size is $1.25 \mathrm{MW}$ at the time for TC, while for the slowest response case (TD equal to $150 \mathrm{~s}$ and 3 operating jet fan pairs) the fire size is $15 \mathrm{MW}$ (half of the peak value). Due to these relatively small HRR values at TC, the dependence of TC-TD on TD is small (the dashed curves in Fig. 12 are almost coincident). This dependence is expected to be more significant for faster developing fires (e.g. pool fires).

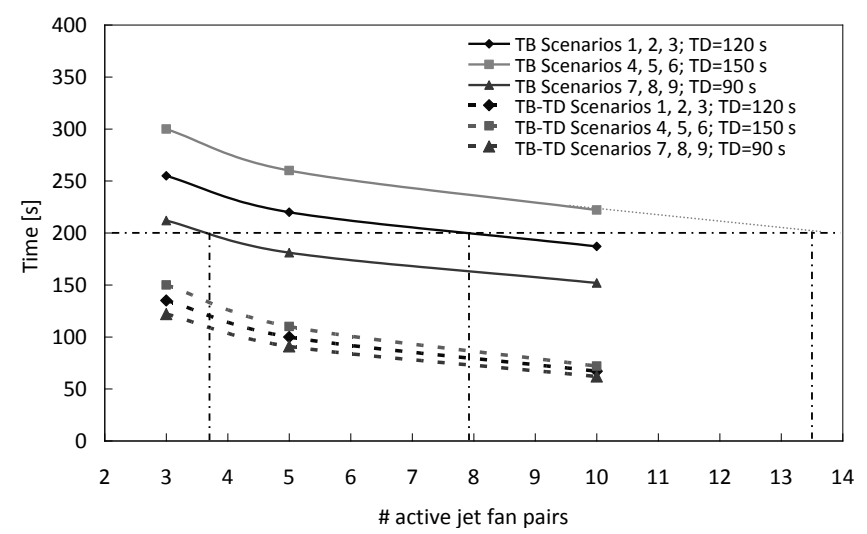

Fig. 13. Dependence of TB-TD (time required to remove back-layering after time to detection) and TB on the number of operating jet fan pairs and TD.

Figure 13 summarizes the dependence of TB and TB-TD on TD and the number of operating jet fan pairs. As it can be seen, the dependence of TB-TD on TD is more significant because, for longer TD, the upstream smoke has travelled longer distances from the seat of the fire by the time the ventilation system is activated (see Fig. 13). This dependence becomes smaller when more jet fans are simultaneously activated given the larger momentum of the ventilation flows in comparison to the buoyancy strength of the plume; indeed, the results tend to converge as the number of operating fans increases.

The results of the transient analysis allow for simultaneous optimization of the ventilation and detection systems. For example, if a fire safety system needs to be designed to cope with a $30 \mathrm{MW}$ fire and to remove back-layering in less than $200 \mathrm{~s}$, the following combined ventilation/detection systems would be equivalent (see Fig. 13):

- 4 jet fan pairs and a detection technology able to detect the fire within $90 \mathrm{~s}$,

- 8 jet fan pairs and a detection technology able to detect the fire within $120 \mathrm{~s}$,

- 14 jet fan pairs and a detection technology able to detect the fire within $150 \mathrm{~s}$. 
The three design options perform equally and thus the final design could be chosen on the basis of logistics, reliability, maintenance and cost reasoning.

\section{CONCLUSIONS}

A novel and fast modeling approach is applied to simulate tunnel ventilation flows during fires. The technique is based on the evidence that there are very long tunnel regions where the flow is fully developed (far field), and relatively short portions showing 3-D flow conditions (near field). The multiscale methodology uses different levels of computational sophistication to model each tunnel region saving computational time without decreasing the numerical accuracy.

A multiscale modeling technique has been applied to simulate the transient response during a fire emergency in a realistic tunnel. A series of ventilation scenarios involving a different number of operating jet fans have been simulated. The effects of the mesh size and location of the 1-D-3-D interfaces have also been investigated. It is shown that the accuracy of the multiscale model is high when compared to the full CFD solution. The transient simulations allow for a complete analysis of the ventilation system response and its interaction with the fire. Information on the time required to reach critical velocity conditions, to remove back-layering could be obtained in a computationally efficient approach. This information is fundamental to develop fire safety strategies in tunnels, to design evacuation procedures and to determine the optimum timing for the activation of fire fighting systems (e.g. water mist or deluge systems). The results confirm the importance of number of operating jet fans and the detection time on the ventilation system response.

A reduction of the required computing time by two orders of magnitude is achieved. The multiscale model has been demonstrated to be a valid technique for the simulation of complex road tunnel ventilation systems. It can be successfully adopted to conduct parametric and sensitivity studies, to design longitudinal and transversal ventilation system, to assess system redundancy and to assess the performance under different hazards conditions. Furthermore, the authors believe that the multiscale methodology represents the only feasible tool to conduct accurate simulations in tunnels longer than few kilometers, when the limitation of the computational cost becomes too restrictive.

\section{ACKNOWLEDGEMENT}

The authors thank the collaboration and support from Dr Ricky Carvel at the University of Edinburgh.

\section{REFERENCES}

[1] Colella, F., Rein, G., Borchiellini, R., Carvel, R., Torero, J., and Verda, V., (2009) Calculation and design of tunnel ventilation systems using a two-scale modelling approach, Building and Environment 44(12): 2357-2367, http://dx.doi.org/10.1016/j.buildenv.2009.03.020.

[2] Colella, F., Rein, G., Borchiellini, R., and Torero, J., (2010) A Novel Multiscale Methodology for Simulating Tunnel Ventilation Flows During Fires, Fire Technology 47(1): 221-253, http://dx.doi.org/10.1007/s10694-010-0144-2.

[3] Van Maele, K. and Merci, B., (2008) Application of RANS and LES field simulations to predict the critical ventilation velocity in longitudinally ventilated horizontal tunnels, Fire Safety Journal 43(8): 598-609, http://dx.doi.org/10.1016/j.firesaf.2008.02.002.

[4] Colella, F., Rein, G., Carvel, R., Reszka, P., and Torero, J., (2010) Analysis of the ventilation systems in the Dartford tunnels using a multi-scale modelling approach, Tunnelling and Underground Space Technology 25(4): 423-432, http://dx.doi.org/10.1016/j.tust.2010.02.007.

[5] Merci, B., (2008) One-dimensional analysis of the global chimney effect in the case of fire in an $\begin{array}{lllll}\text { inclined } & \text { Fire } & \text { Safety } & \text { 376-389, }\end{array}$ http://dx.doi.org/10.1016/j.firesaf.2007.09.003.

[6] Versteeg, H.K. and Malalasekera, W., An introduction to computational fluid dynamics, the finite volume method, Longman scientific \& technical, Harlow, Essex, England, 1995, pp. 67-75. 
[7] Rodi, W., "Turbulence models and their application in hydraulics," University of Karlsruhe, Karksruhe, Germany, 1980.

[8] Woodburn, P. and Britter, R.E., (1996) CFD simulations of a tunnel fire-Part I, Fire Safety Journal 26(1): 35-62, http://dx.doi.org/10.1016/0379-7112(96)00018-5.

[9] Woodburn, P. and Britter, R.E., (1996) CFD simulations of a tunnel fire-Part II, Fire Safety Journal 26(1): 63-90, http://dx.doi.org/10.1016/0379-7112(96)00019-7.

[10] Oka, Y., and Atkinson, G.T., (1995) Control of smoke flow in tunnel fires, Fire Safety Journal 25(4): 305-322, http://dx.doi.org/10.1016/0379-7112(96)00007-0.

[11] Vauquelin, O. and Wu, Y., (2006) Influence of tunnel width on longitudinal smoke control, Fire Safety Journal 41(6): 420-426, http://dx.doi.org/10.1016/j.firesaf.2006.02.007.

[12] Karki, C.K., Patankar, S.V., Rosenbluth, E.M., and Levy, S.S., "CFD model for jet fan ventilation systems," $10^{\text {th }}$ International Symposium on Aerodynamics and Ventilation of Vehicle Tunnels Principles, Analysis and Design, BHR Group Limited, 2000, pp. 335-380.

[13] Vega, M., Diaz, K., Oro, J.M, Tajadura, R., and Morros, C., (2008) Numerical 3D simulation of a longitudinal ventilation system: Memorial Tunnel case, Tunnelling and Underground Space Technology 23(5): 539-551, http://dx.doi.org/10.1016/j.tust.2007.10.001.

[14] Colella, F., "Multiscale analysis of tunnel ventilation flows and fires", PhD thesis, Politecnico di Torino, Dipartimento di Energetica, 2010.

[15] Quarteroni, A. and Valli, A., Domain Decomposition Methods for Partial Differential Equations, Clarendon Press, Oxford, 1999, pp. 296-320.

[16] Formaggia, L., Gerbeau, J.F., Nobile, F., and Quarteroni, A, (2001) On the coupling of 3D and 1D Navier-Stokes equations for flow problems in compliant vessels, Computer Methods in Applied Mechanics and Engineering 191(6-7): 561-582, http://dx.doi.org/10.1016/S0045-7825(01)00302-4

[17] Wu, Y., Bakar, M.Z.A., (2000) Control of smoke flow in tunnel fires using longitudinal ventilation systems - a study of the critical velocity, Fire Safety Journal 35(4): 363-390, http://dx.doi.org/10.1016/S0379-7112(00)00031-X

[18] Heselden, A.J.M, "Studies of Fire and Smoke Behavior Relevant to Tunnels," Current Paper CP66/78, Building Research Establishment, 1978.

[19] Carvel, R., "Design fires for tunnel water mist suppression systems," $3^{\text {rd }}$ International Symposium on Tunnel Safety and Security, SP Fire Technology, 2008, pp. 141-148.

[20] Koffmane, G. and Hoff, H., "More than Just Fire Detection: Fibre Optic Linear Heat Detection (DTS) enables Fire Monitoring in Road- and Rail-Tunnels," $4^{\text {th }}$ International Symposium on Tunnel Safety and Security, SP Fire Technology, 2010, pp. 525-528.

[21] Rogner, A, "Safety and Reliability of Fire Detection Systems in Road Tunnels," $4^{\text {th }}$ International Symposium on Tunnel Safety and Security, SP Fire Technology, 2010, pp. 561-564.

[22] Liu, Z.G., Kashef, A., Lougheed, G.D., Crampton, G., Ko, Y., and Hadjisophocleous, G., "Parameters affecting the performance of detection systems in road tunnels," $13^{\text {th }}$ International Symposium on Aerodynamics and Ventilation of Vehicle Tunnels, BHR group Limited, 2009, pp. 389-402. 